\title{
Organizational Information Dissemination Within Collaborative Networks Using Digital Communication Tools
}

\author{
Cristelia Hinojosa \\ Nova Southeastern University \\ ch1244@nova.edu
}

\author{
Simon Cleveland \\ Nova Southeastern University \\ sc1674@nova.edu
}

\begin{abstract}
Knowledge transfer among employees remains a challenge for many organizations. With the increased adoption rate of corporate social media collaboration technologies, there is an urgent need to determine the factors that enhance information diffusion among employees.

The current study leverages prior research on social media collaboration performed in the public domain to determine whether the dimensions of tie strength have any effect on information diffusion among corporate users of social media technologies. Analysis of 511 Lync messages related to a training topic at a mid-sized South Central corporation was performed. The preliminary results demonstrated that the dimensions of tie strength (intimacy, structural, and social) were highly correlated to the number of messages among users. Moreover, regression analysis showed that three quarters of the messages were also predicted by the dimensions of tie strength.
\end{abstract}

\section{Introduction}

Information flow is necessary for maximizing profits and performance within an organization [7]. Sharing knowledge is essential to an organization's ability to gain a competitive advantage [22].

The people within an organization possess critical knowledge, and there is a need to motivate these individuals to share their knowledge and make it readily available to those who require access to it. Including employees' knowledge in operations adds relevance to the organization as knowledge sharing improves organizational change and has both economic and social value [24]. However, transferring knowledge between organizations is complex due to dispersal coordination and high costs, and managers continue to experience difficulty leveraging employees’ knowledge [8], [24].

Promoting knowledge transfer has long been a challenge for organizations who rely on knowledge for decision making [26]. An organization's knowledge creation, innovation, and competitiveness are hindered when employees are not motivated to share knowledge [5]. While organizations have implemented knowledge management systems designed to facilitate knowledge transfer within an organization, these organizations continue to face difficulty motivating employees to contribute knowledge [31].

Recently, companies have begun to invest in collaborative social media systems to facilitate the knowledge sharing process, yet such systems often result in a significant investment for an organization. As a result, organizations need to determine if the expense of purchasing and implementing a collaboration system is worth the investment and understand if it will be effective in spreading knowledge.

Extant studies show that relationships among individuals are critical for the development of an information transfer process [3], [9], [12], [18], [32]. An advantage of social interaction is the access to knowledge that it provides [14], [8], [33]. However, all relationships should not be treated equally, as individuals maintain ties that affect how they interact with other members of the same network [20]. As a result, understanding relational ties is essential for the development of efficient dissemination of information [20].

Tie strength is a relationship factor that has been used to measure social influence within a network [2] [3]. Moreover, tie strength's impact on information diffusion within public online social networks have been measured quantitatively by collecting data through digital communication tools [3], [11], [15], [16]. However, while recent studies have measured 
the influence of tie strength within public social networks, there is a dearth of research on measuring tie strength's impact on information diffusion within a collaborative social network.

Collaborative networks are types of social networks that are formed by members who work together to achieve specific goals [23]. These types of networks have significantly different characteristics from the public social networks, since they are subject to organizational constraints that make the knowledge sharing process different from that of a public social network [7], [23]. As a result, there is a need to statistically investigate the social influences of utilizing a unified communication tool to share knowledge within a private collaborative social network.

The current study proposes to narrow the existing research gap by investigating how the personal characteristics of tie strength influence information dissemination within a corporate collaborative social network in an organization that uses online social network technology. It performs a preliminary analysis of conversations among corporate users of a social network system at a mid-size South Central company. Using the proxies that have been utilized to measure tie strength within public social networks, the study aims to determine how tie strength influences the effects of information dissemination within a corporate collaborative social network.

The rest of the paper is structured as follows. First, a literature review of the existing research on the diffusion of information within public social networks is performed. The dimensions of tie strength are identified, and the concept of corporate collaborative social network is defined. Next, an analysis of a sample of 511 conversations among corporate collaborative network participants is performed. The paper concludes with a call for further research on the role of the dimensions of tie strength and the information diffusion within private collaborative networks.

\section{Literature review}

Knowledge provides economic value to an organization. As a result, companies are investing in methods for transferring information among members of their organizational network in order to retain knowledge and increase their competitive advantage.

Previous literature provides an increased understanding about the factors that contribute to knowledge sharing within an organization. For example, Casmir et al. [5] indicated that a collaborative culture within an organization promotes knowledge sharing. Mura et al. [24] discussed the relationship between trust within an organization and producing an environment in which knowledge sharing is conducive. These findings support the idea that knowledge sharing is a social process that requires social interactions between knowledge contributors and receivers. The literature validates the relevance of further observation of social factors that contribute to knowledge sharing in an organizational environment.

\subsection{Knowledge sharing within online social networks}

Knowledge sharing is a social process that is affected by social motivational factors [19]. Interpersonal relations and the resources that are generated through social networks are vital to interfirm knowledge transfer [14]. Social cohesion motivates individuals to invest in knowledge sharing efforts [8]. Personal networks are composed of people with whom a person maintains contact, including partners, customers, suppliers, and family members [8]. Public social networks focus on social interactions and social information exchange [23]. The structural properties of a social network are exhibited in an online network [16]. Recent studies regarding information distribution within social networks have focused on public social networks, which commonly use social media platforms such as Facebook [3], [10], [15], [20].

An individual's learned knowledge is subject to external relationships. Casmir et al. [5] argued that knowledge sharing is facilitated by organizations that promote trust. Social influence is a determining factor of what people adopt and when they adopt it [19]. Lin and Lo [19] identified that there remains a need to investigate how to motivate employees to share knowledge, as employees within an organization possess critical knowledge that is not always easily available to those that need access. While the motivational factors of knowledge sharing have been widely researched, gaps exist in taking into consideration the relationship between a person's position in a social network and the degree in which a person controls information [19].

Barker [4] performed a study in which the use of a discussion forum was tracked to determine its role in knowledge creation and sharing. The research proposed a theoretical framework based on the components of content, communication, and consumer for measuring knowledge management within an online discussion forum. It was discovered that within an online discussion forum, knowledge creation and sharing is best supported when it is 
being managed by an expert within the organization and the discussion forum has expert intervention.

Relationships are an influencing factor of knowledge sharing. The similarities between a public social and a collaborative network can allow for research concerning knowledge sharing to be applied to both networks. Research regarding relationships within a social network can be utilized to understand the similarities of knowledge sharing within a collaborative social network. Since tie strength is a relationship factor, it can be used to understand the knowledge sharing process within a network. The proxies used to determine tie strength within a social network can be measured within a collaborative social network.

\subsection{Collaborative networks}

Reviewing literature regarding relationship factors within an organization provided an understanding of how to produce a more collaborative environment in which knowledge sharing can be increased. Collaborative social networks can be on the web or within the private sector, but because they are included in organizational networks, they are subject to organizational constraints. There are drastic differences in how knowledge flows in a collaborative social network as opposed to a public social network due to the random factors involved in human decision making [23]. Collaborative networks require adequate information flow to overcome organizational complexities [7]. Public social networks focus on social interactions and social information exchange, and information is first derived at a particular source and then spread by members of the network forwarding activities [23]. Maio et al. [23] explained that in a public social network, the information begins to lose its value at the same time as the forwarding activities begin to diminish. In contrast, information flow in a collaborative network is driven by specific tasks and the expertise of the members assigned to the network as tasks in collaborative networks travel along long sequences of steps before completed [23].

Durugbo [7] performed a comparative, multiple case study on six industrial firms to investigate the flow of information within a collaborative networked organization. It was concluded that the flow of information could be enhanced within an industrial firm when procedures and policies are prioritized. Moreover, the study showed that within collaborative networks, information is built from relationships that allow firms to collaborate.

Collaborative networks are enhanced with the information flowing through industrial firms.
Information is built from relationships which allow firms to collaborate. For example, Rejeb-Khachlouf et al. [8] concluded that dense networks contribute to a faster diffusion of information. Chui et al. [16] validated that structural properties of a social network are exhibited in an online network and that tie strength is an influencing factor of certain network behavior.

Since there are vast differences between social networks and collaborative networks, the studies that examine knowledge sharing should be replicated within each type of network. The process of how research is conducted within a social public network can be applied to collaborative social networks that also utilize technology platforms for transferring information.

\section{Tie strength and information diffusion}

Tie strength is a relationship factor that has been used to measure social influence within a network [2] [3]. Tie strength is the nature of ties between an individual and other members of the same network and characterizes the closeness and interaction frequency of a relationship between two people [12]. Granovetter [12] proposed that tie strength can be measured by evaluating a combination of four dimensions: amount of time, emotional intensity, intimacy, and reciprocal services.

This quantifiable characteristic of a social network can be measured to determine the link between nodes [20]. It has often been assumed that relationships within a network are equal, but that fails to consider that people maintain relationships with varying tie strength [16]. The role of ties within a network varies depending on the network's strength, and different tie strengths affect the social structure differently [16]. Individuals with strong ties have greater similarities and share more intimate information, so treating all relationships as equal degrades predictive modeling [20]. Verifying the frequency and duration of interactions allows a better method of determining tie strength [20].

Luarn and Chiu [20] performed a correlation study to develop a predictive model to quantitatively measure tie strength within social network sites by examining user profile similarities and user interactions of passing data on Facebook as well as user responses to an online questionnaire. Subjective tie strength was measured from the online questionnaire while objective tie strength was perceived from the data set that included the participant's interaction records to determine the strength of the friendships within Facebook. 
Bakshy et al. [3] utilized an experimental method to explore social media's role in information diffusion by investigating the use of a social digital collaboration tool and the influence of strong and weak ties. Using Facebook as a platform, the researchers randomized whether participants were shown information about their friends' sharing behavior and measured how this tie strength influenced their likelihood of sharing a Uniform Resource Locator (URL) with others. They discovered that subjects who receive signals about friends' sharing behaviors have a higher probability of sharing information than those that are not exposed to this information.

Similar to Bakshy et al. [3], Aral and Walker [2] conducted an experiment by randomizing data within a networked environment and performing statistical analysis. Making use of automated notifications, the researchers randomly delivered information to random peers concerning the senders' personal attributes and use of the application. The study concluded that people are more influenced by peers with whom they are embedded. The individual effects of each measure of tie strength upon influence was identified.

\subsection{Tie-strength measurements}

Research concerning tie strength continues to use the original dimensions proposed by Granovetter [12], which consist of four dimensions to define the strength of two people's relationship: amount of time, intimacy, emotional intensity, and reciprocal services. While Granovetter [12] proposed the seminal theory defining tie strength that has been used for decades, he indicated in his study that the empirical measurement methods for measuring tie strength were to be determined by future studies. Adali et al. [1], Chiu, Chen, Joung, and Chen [16], Gilbert and Karaholios [11], Gupte and Eliassi-Rad [13], He, Zhang, and Ji [15], Luarn and Chui [20], Marsden and Campbell [21], Petroczi et al. [27], and Steffes and Burgee [28] have conducted studies that adopt the criteria of Granovetter [12] to construct models for measuring the various dimension of tie strength. Granovetter's [12] work has been interpreted, and some current literature now includes a total of seven dimensions for examining tie strength: duration and frequency of contact, intimacy, intensity, emotional support, reciprocal services, structural variables, and social distance [10]. Each dimension can be evaluated and used to calculate the total strength of a tie.

While the original measurement models assumed physical interaction as the definition stated, the new models for measuring public social networks consider social media sites and assume that interaction takes place online [2], [3], [13], [15]. As such, these models have focused on measuring tie strength through characteristics that can be captured through social media platforms. Although studies do not exist that provide a model for measuring tie strength in a collaborative network, measurements of tie strength within a public network will be analyzed to determine how the models can be applied to a collaborative network.

Chui et al. [16] performed a correlation study to develop a model for quantitatively measuring the role of tie strength within an online social network by using a crawling agent to digitally collect data within an online blog. Tie strength was quantified as the frequency of interactions between two actors, which was the number of comments a user posted for another user. The "friends" list within the blog was also considered, and if one actor considered another a "friend" it was categorized as a friendship relationship. The study determined that the structural properties of a social network are exhibited in an online network, and tie strength may be the cause of certain network behavior. Within an online social network, there is a strong association between tie strength and reciprocity and tie strength and transitivity.

\section{Proposed study}

To determine how tie strength influences the effects of information dissemination within a collaborative social network, this paper proposes a causal modeling study. The independent variables will be the multiple dimensions of tie strength, which will be measured by the tie strength proxies that have been identified within the literature [10], [20]. The dependent variable will be information dissemination, which will be measured by tracking the amount of information transmitted via a collaboration tool within an organization. A live environment that is using multiple modalities of Microsoft Lync as a collaborative tool to discuss training topics will be observed. Lync communication logs will be monitored to determine how the collaboration tool is used to disseminate information related to specific training topics.

Any information related to the training topics that is passed between Lync users via the collaboration tool will be tracked by contextual analysis of messages sent within the system. This data will be quantified to determine how information is disseminated via the collaboration tool. The goal of 
the study will be to determine if tie strength influences information dissemination, as measured by monitoring the information sent via the collaboration tool that supports knowledge transfer within an organization.

\subsection{Hypotheses}

The following hypotheses are proposed for the study:

H1-1: The stronger the tie strength as demonstrated by intimacy, the more likely the diffusion of information. H1-2: The stronger the tie strength as demonstrated by structural variables, the more likely the diffusion of information.

Employees within the same professional occasional group can be considered members of a community of practice, which are informal groups with shared expertise. Members of communities of practice often have shared memory and knowledge, which allows information to be openly diffused within their community [6]. As such, it will be hypothesized that members with the same job classification will have stronger tie strength, which will increase the likelihood of information diffusion within their group. Therefore, it is proposed that:

H1-3: The stronger the tie strength as demonstrated by social distance, the more likely the diffusion of information.

\subsection{Research environment}

To extend the work of Bakshy et al. [3] who researched the effects of social characteristics on information diffusion, the proposed study will monitor a collaborative network within an organizational environment rather than a social communication network. Lync is an online collaboration technology that contains multiple modalities with various levels of richness, including instant messaging, groupware, video conferencing, screen sharing, audio conferencing, chat rooms, and file transfer. Lync will be utilized as the platform for collecting data regarding content sharing. Data will be collected from a single, live business environment within the United States that has been using Lync as an online collaborative network within their organization for at least 12 months. The use of the collaboration tool will be monitored by gathering data from system usage and communication logs to determine the effects of tie strength on the information that is being diffused through the system.

Within this research environment, Lync has been made available to employees who are distributed across the organization and have various roles, responsibilities, and relationships throughout the company's multiple business divisions. Within the company being monitored, Lync is being used to complement training sessions. Subject matter experts throughout the company are sharing their knowledge by holding training sessions concerning particular topics in their field of expertise. Participants attend the training in-person or through the Lync video conferencing feature. The training is supplemented through the use of Lync chat rooms that have been created by meaningful users to continue discussing the training topics between training sessions. The chat rooms allow the training participants to stay connected and continue exchanging information relevant to the training topic.

\subsection{Participants}

Participants will be selected from a population of employees who utilize Lync within an organization and are considered meaningful users of the system. The company's data logs that record Lync system usage will be evaluated to determine people that send at least 10 messages per month, and these users will be classified as meaningful users as opposed to those that simply have access and are not making meaningful use of the system. For the purpose of this study, a relationship will be defined as the connection between a meaningful Lync user. All meaningful users will be considered to have a relationship, whether or not they communicate with each other using the Lync system, because they are all related to the same organization and are all members of the same collaborative network.

Chat rooms that have been created to supplement training sessions and have been in existence for at least one month will be monitored for the purposes of this study. The chat rooms selected will be sharing information about a single topic and will have at least 10 members that are dispersed geographically throughout the company. The chat rooms monitored during the study will make use of multiple modalities of the Lync system, including integration with other systems. A subject matter expert of the training topic will be included in each chat room in accordance with the research of Barker [4] that determined knowledge sharing is best supported within an online environment when there is intervention by an expert. The members of each chat room will be used as participants of the study. 


\subsection{Variables, data collection and causal modeling}

Three dimensions of tie strength will be used as independent variables to evaluate social influence. Since tie strength is a quantifiable characteristic of a social network, the proposed study will utilize this multidimensional construct to determine the influence of social relationships on information dissemination [20]. Ties capture the function of relationships based on statistical properties of communication patterns. Tie strength will be used as a predictor of information dissemination. Tie strength consists of dimensions that will be used as latent variables. The dimensions of tie strength cannot be directly measured. However, each of the tie strength dimensions contain proxies that can be used as observed variables. These proxies are behaviors that can be observed and have been used in previous studies to measure tie strength.

The identification of the multiple dimensions of tie strength will be based upon the work of Gilbert and Karaholios [11] and Luarn and Chui [20], who captured user information from social networks to quantify the strength of a tie within a social network. The proxies utilized by Gilbert and Karaholios [11] and Luarn and Chui [20] were based upon characteristics that could be captured from a public social network rather than surveys, and these proxies best reflect the type of data that can be captured from a collaborative network.

Granovetter [12] was the originator of the tie strength theory, which included four dimensions to define the strength of two people's relationship. Tie strength is defined by Granovetter [12] as "a combination of the amount of time, emotional intensity, intimacy, and reciprocal services devoted to a relationship” (p. 1361).

Original tie strength proxies only classified face to face communication, and recent research has evolved the proxies to accommodate purely online communication that takes place in a public social network. With the emergence of online social networks, recent literature has dissected Granovetter's [12] theory to begin forming their own interpretations and developing models for measuring tie strength that fits the online social network environment. Krackhardt [17] measured tie strength by using the dimensions of duration and frequency of contact and reciprocal services. He [15] measured tie strength using the dimensions of emotional intensity and intimacy. Gilbert and Karaholios [11] measured tie strength using the dimensions of structural variables and social distance. Gilbert and Karaholios [11] developed models that were generated by the aspect of tie strength that was most measurable within an online environment. These new models allow measurement of tie strength through pure online interaction, rather than physical interaction that was originally theorized.

Three dimensions of the independent variable tie strength will be used as a predictor variable and measured to determine the strength of relationships: intimacy, structural variables, and social distance. The multiple dimensions of tie strength will be measured based upon previous studies that have measured multiple tie strength proxies. The work of recent researchers that measure tie strength within an online environment will be used as a basis for defining proxies that should be used within a collaborative environment. The relationships between the participants will be determined by cross referencing the organization's human resource database and the communication logs within Lync. The proposed study will also model the work of Wang et al. [30] who cross-referenced information from an organization's database in order to identify employee personal characteristics.

Each proxy of tie strength will be a ratio variable, because the variables of intimacy, structural variables, and social distance are limited to the number of possible values. Each of the independent variables will be evaluated by five elements, and the number of elements that are present for each indicator will be counted and used to form a ratio for each variable. The ratio will be represented by numbers (0-5) that represent the actual number of indicators that are present. Each of the five indicators will be worth one point in the ratio. The number of indicators present will indicate the ratio of the independent variable, where an indicator of ' 0 ' will represent that no indicators are present. For example, a ratio of ' 0 ' represents that zero indicators are present and a ratio of ' 5 ' represents that five indicators are present. These ratio variables will be included as independent variables in the regression analysis.

4.4.1. Independent variables. Gilbert [10] utilized proxies such as days since last communication and the number of intimacy words used within a conversation. Bakshy et al. [3] identified the frequency of private online communications between users. For the purpose of this study, the data from other company systems including the Human Resource system will be cross referenced to identify the five different factors that will be used to form a ratio to categorize the intimacy variable. The five elements for intimacy will include: (1) determining if two people have worked in the same department, (2) 
determining if two people have exchanged a private Lync communication message, (3) determining if two people are currently working on the same project, (4) determining if two people are related, (5) determining if two people are currently part of the same Lync group. The proxy of intimacy will be a ratio variable.

Structural variables have been identified in previous research by determining the number of mutual friends and relations between two individuals such as family member, classmate, friend, or attending the same college. This data is more easily determined within a social network platform in which users identify this characteristic within their profiles. Five factors will be identified and used to form a ratio to categorize the structural variable category. The five elements for structural variables will include: (1) determining if two people have the same job classification, (2) determining if two people have ever worked on the same project, (3) determining if two people report to the same direct supervisor, (4) determining if two people work in the same building, (5) determining if two people report to the same geographic location. The proxy of structural variables will be a ratio variable.

Social distance has been measured by identifying commonalities in gender, age, hometown, degree of education, race, and language status. For the purpose of this study, information that is captured within an employee's records and linked to the system profile can be utilized for this measurement. The five elements for social distance will include: (1) determining if two people are the same gender, (2) determining if two people are within the same age range, (3) determining if two people reside within the same city, (4) determining if two people are the same ethnicity, (5) determining if two people have the same occupational prestige. The proxy of social distance will be a ratio variable.

4.4.2. Dependent variable. Information diffusion will be a ratio variable derived by counting the number of relevant communications recorded for their participant their connections across all communication modes. The output variable of interest will be information diffusion within an organization as measured by tracking content that is sent within the Lync system and determining how information provided regarding the training sessions are sent through the Lync system. Any message that contains references to information pertaining to the training topics will be considered information diffusion. Information diffusion, the dependent variable, will be measured by quantifying the number of references made to information about the training topics via the Lync tool.
4.4.3. Causal modeling. The research will be performed as causal modeling, which follows the process of Aral and Walker [2], Bakshy et al. [3], and Steeg and Galstyan [29] to identify how the variables of tie strength influence information dissemination within a social network. A causal model allows for multiple causality, which is appropriate for this study in which the multiple dimensions of tie strength will be tested for their effect on the dependent variable. Causal modeling will have the ability of determining the combined impact of the dimensions of tie strength on information dissemination. This study will introduce a new dimension to Aral and Walker's [2] and Bakshy et al. [3] studies by employing causal modeling to determine the influence of social ties on information diffusion within an organization.

The causal model will be based on structural equation modeling and analyzed by regression techniques. Regression models are used to estimate the numerical effect of one variable upon another. This study will utilize regression analysis to examine the interaction variables and tie strength. Structural equation modeling (SEM) using regression analysis will be utilized to determine the impact of each tie strength dimension on information diffusion within a collaborative network.

After the proxies of tie strength are utilized to determine the tie strength between each of the individuals, and use of the collaboration technology is used to measure information diffusion, SEM will be applied to measure the influence of each tie strength indicator on information diffusion. A minimum of 10 relationships should be measured for each parameter for SEM. A minimum of 600 relationships will be measured, which will include relationships between the participants within the Lync system chat rooms.

The tie strength relationship between each participant of the study will be examined. For each of the categorical independent variables of tie strength, the researcher will use a category of 0-5 depending on the number of indicators that are present for each variable. A ' 0 ' category will indicate that no indicators are present for that variable. Intimacy is measured by shared relationships between two people with five elements identified to determine the ratio of this variable. Structural variables are measured by identifying shared affiliation or networks between two people, and five elements have been identified to determine the ratio for this variable. Social distance has been measured by identifying commonalities employee characteristics, and five elements have been identified to determine the ratio for this variable. 
For each relationship within the chat room, the data gathered regarding use of the entire Lync system will be used to count the number of times that each relationship made reference to information relevant to the training topic. Communication occurring within any of the Lync modalities will be used to measure the dependent variable, information dissemination. To determine if a message contains information related to the training session, a database will be created consisting of words and phrases that are consistent with the training topic for each chat room. For example, if the class is teaching Spanish, any reference to the Spanish material learned and references to the class will be included in the database. Visual content analysis along with the use of an E-discovery tool will be utilized to analyze the content of messages exchanged between the participants to find relevant references to the training topics. Regression analysis will be used to determine the impact of tie strength on information diffusion.

SEM with ratio variables will be used to analyze the data. Numerical integration will be used in which the number of dimensions will be the number of latent variables. The seminal work of Muthen [25] will be used for analyzing the data using SEM. The thresholds will be estimated to determine the maximum likelihood of using univariate data. The correlations will then be estimated from the binary data. Weighted least squares will be used to estimate the SEM model. SEM will allow the researcher to simultaneously test a set of regression equations. Regression analysis will be performed to determine each of the latent variables' impact on information diffusion. This will be performed by entering the data gathered regarding the number of relationships that passed information regarding the training topics for each dimension of the independent variable into the software analysis tool.

\section{Preliminary study}

In order to validate the relevancy of the study and justify further research, preliminary Lync log data was gathered from the servers of a mid-size South Central company. Information diffusion was measured by counting the conversation messages related to corporate training among the Lync users.

A sample of 736 messages among users was collected for the preliminary study. A single coder reviewed each of the messages and counted only those related to the corporate training session. The coding eliminated 225 of the messages, leaving a total of 511 training-related messages within 21 relationship connections.
Next, the coder rated the three dimensions of tie strength (intimacy, structural, and social) on a scale of $0-3$ for each conversation initiator. The three factors of intimacy included in the preliminary study were: (1) determining if two people have worked in the same department, (2) determining if two people have exchanged a private Lync communication message, and (3) determining if two people are currently working on the same project. The three structural variable factors included in the preliminary study were: (1) determining if two people have the same job classification, (2) determining if two people report to the same direct supervisor, (3) determining if two people work in the same building. The three social distance factors included in the preliminary study were: (1) determining if two people are the same gender, (2) determining if two people are within the same age range, and (3) determining if two people are the same ethnicity. The results are provided in Table 1, which identifies the number of messages and dimensions rating of each conversation starter. Table 2 provides the descriptive statistics of the results.

To determine correlation between tie strength and the total number of messages, the three dimensions were averaged to produce the values for the tie strength variable. Pearson correlation coefficient was calculated between tie strength and total number of messages. The results demonstrated that the two variables were correlated $(r=.87)$.

A regression analysis was also performed to determine whether tie strength can predict the number of messages. The results showed that three quarters of the total conversations were predicted by the tie strength $\left(\mathrm{R}^{2}=.76, \mathrm{~F}=60.604, \mathrm{p}<.001\right)$.

Table 1. Messages and dimensions rating

\begin{tabular}{|c|c|c|c|c|c|}
\hline From & To & $\begin{array}{c}\text { Total \# } \\
\text { Messages }\end{array}$ & $\begin{array}{c}\text { Intimacy } \\
(0-3)\end{array}$ & $\begin{array}{c}\text { tructur } \\
(0-3)\end{array}$ & $\begin{array}{r}\text { Socia } \\
(0-3)\end{array}$ \\
\hline AA & $A B$ & 96 & 3 & 2 & 1 \\
\hline$A A$ & $A C$ & 29 & 1 & 1 & 1 \\
\hline AA & $A D$ & 28 & 1 & 1 & 0 \\
\hline$A A$ & $A E$ & 26 & 2 & 0 & 1 \\
\hline AA & AF & 28 & 2 & 1 & 0 \\
\hline$\overline{A A}$ & AG & 134 & 3 & 2 & 2 \\
\hline$A B$ & $A C$ & 6 & 0 & 1 & 0 \\
\hline$A B$ & $A D$ & 6 & 1 & 1 & 0 \\
\hline$A B$ & $\mathrm{AE}$ & 2 & 1 & 0 & 0 \\
\hline$A B$ & AF & 5 & 0 & 1 & 0 \\
\hline$A B$ & AG & 61 & 2 & 2 & 1 \\
\hline$A C$ & $A D$ & 13 & 1 & 1 & 1 \\
\hline$A C$ & $A E$ & 10 & 1 & 0 & 2 \\
\hline$A C$ & AF & 11 & 0 & 1 & 1 \\
\hline$A C$ & $A G$ & 7 & 0 & 1 & 2 \\
\hline$A D$ & $\mathrm{AE}$ & 7 & 0 & 1 & 1 \\
\hline$A D$ & AF & 10 & 0 & 1 & 2 \\
\hline$A D$ & $A G$ & 6 & 0 & 1 & 1 \\
\hline$A E$ & AF & 8 & 0 & 0 & 1 \\
\hline $\mathrm{AE}$ & $A G$ & 6 & 1 & 0 & 2 \\
\hline AF & AG & 12 & 2 & 1 & 1 \\
\hline
\end{tabular}


Table 2. Descriptive statistics

\begin{tabular}{|l|c|c|c|}
\hline \multicolumn{1}{|c|}{ Variable } & N & Mean & SD \\
\hline Total \# Messages & 21 & 24.33 & 33.55 \\
\hline Intimacy & 21 & 1.00 & 1.00 \\
\hline Structural & 21 & 0.90 & 0.62 \\
\hline Social & 21 & 0.95 & 0.74 \\
\hline
\end{tabular}

\section{Conclusion and future research}

Determining tie strength's role in information dissemination can allow an organization to utilize this relationship factor to increase information flow, resulting in strengthened employee collaboration and increased collective knowledge. This preliminary study begins to address the research question of how tie strength influences the effect of information dissemination within a collaborative social network. The preliminary data indicates that stronger tie strength has a positive effect on information dissemination. The results of the preliminary findings justify the need to conduct the full research outlined in this article, including the evaluation of a larger sample size, which will assist in validating these results.

Furthermore, the preliminary results support the hypothesis that stronger tie strength resulted in increased information dissemination as the relationships that indicated a stronger tie strength shared more information within the collaboration system. Gathering a larger set of data will allow the researchers to utilize SEM to determine the effect of tie strength on information dissemination.

One limitation of the study is its generalizability since the data was collected within one business environment within the United States. Future studies could include data collection across multiple organizations to compare the findings. Another limitation is the study's lack of consideration of the possible effect that various modalities within the collaborative system have on information transfer. A future study can incorporate the multiple modalities as a moderating variable to test their effect on the dependent variable.

In conclusion, organizations can leverage this study to determine the value of their collaboration systems and whether efforts should be conducted to promote tie strength as a mechanism for increasing information sharing among employees. Depending on the results, organizations may decide that the efforts required to implement and maintain a collaboration system are not justified if the amount of information disseminated is determined to be insignificant.

\section{References}

[1] S. Adali, F. Sisenda and M. Magdon-Ismail, Actions speak as loud as words: Predicting relationships from social behavior data, Proceedings of the 21st international conference on World Wide Web, ACM, 2012, pp. 689-698.

[2] S. Aral and D. Walker, "Tie strength, embeddedness, and social influence: A large-scale networked experiment", Management Science, 60 (2014), pp. 1352-1370.

[3] E. Bakshy, I. Rosenn, C. Marlow and L. Adamic, The role of social networks in information diffusion, Proceedings of the 21st international conference on World Wide Web, ACM, 2012, pp. 519-528.

[4] R. Barker, "Management of knowledge creation and sharing to create virtual knowledge-sharing communities: a tracking study", Journal of Knowledge Management, 19 (2015), pp. 334-350.

[5] G. Casimir, K. Lee and M. Loon, "Knowledge sharing: influences of trust, commitment and cost", Journal of Knowledge Management, 16 (2012), pp. 740-753.

[6] M. Del Giudice, M. R. Della Peruta and V. Maggioni, "A model for the diffusion of knowledge sharing technologies inside private transport companies", Journal of Knowledge Management, 19 (2015), pp. 611-625.

[7] C. Durugbo, "Managing information for collaborative networks", Industrial Management \& Data Systems, 114 (2014), pp. 1207-1228.

[8] A. Fayolle, N. Rejeb-Khachlouf, L. Mezghani and B. Quélin, "Personal networks and knowledge transfer in inter-organizational networks", Journal of Small Business and Enterprise Development, 18 (2011), pp. 278-297.

[9] N. E. Friedkin, "Information flow through strong and weak ties in intraorganizational social networks", Social networks, 3 (1982), pp. 273-285.

[10] E. Gilbert, Predicting tie strength in a new medium, Proceedings of the ACM 2012 conference on Computer Supported Cooperative Work, ACM, 2012, pp. 1047-1056. [11] E. Gilbert and K. Karahalios, Predicting tie strength with social media, Proceedings of the SIGCHI conference on human factors in computing systems, ACM, 2009, pp. 211-220.

[12] M. S. Granovetter, "The strength of weak ties", American journal of sociology (1973), pp. 1360-1380.

[13] M. Gupte and T. Eliassi-Rad, Measuring tie strength in implicit social networks, Proceedings of the 4th Annual ACM Web Science Conference, ACM, 2012, pp. 109-118.

[14] M. T. Hansen, "The search-transfer problem: The role of weak ties in sharing knowledge across organization subunits", Administrative science quarterly, 44 (1999), pp. 82-111.

[15] Y. He, C. Zhang and Y. Ji, Principle features for tie strength estimation in micro-blog social network, Computer and Information Technology (CIT), 2012 IEEE 12th International Conference on, IEEE, 2012, pp. 359367.

[16] T. Hui-Ye Chiu, C.-C. Chen, Y.-J. Joung and S. Chen, "A study of blog networks to determine online social 
network properties from the tie strength perspective", Online Information Review, 38 (2014), pp. 381-398.

[17] D. Krackhardt, "The strength of strong ties: The importance of philos in organizations", Networks and organizations: Structure, form, and action, 216 (1992), pp. 239.

[18] D. Z. Levin and R. Cross, "The strength of weak ties you can trust: The mediating role of trust in effective knowledge transfer", Management Science, 50 (2004), pp. 1477-1490.

[19] S.-W. Lin and L. Y.-S. Lo, "Mechanisms to motivate knowledge sharing: integrating the reward systems and social network perspectives", Journal of Knowledge Management, 19 (2015), pp. 212-235.

[20] P. Luarn and Y.-P. Chiu, "Key variables to predict tie strength on social network sites", Internet Research, 25 (2015), pp. 218-238.

[21] P. V. Marsden and K. E. Campbell, "Measuring tie strength", Social forces, 63 (1984), pp. 482-501.

[22] G. Martín-de Castro, P. López-Sáez, M. DelgadoVerde, M. J. Donate and F. Guadamillas, "Organizational factors to support knowledge management and innovation", Journal of Knowledge Management, 15 (2011), pp. 890914.

[23] G. Miao, S. Tao, W. Cheng, R. Moulic, L. E. Moser, D. Lo and X. Yan, Understanding task-driven information flow in collaborative networks, Proceedings of the 21st international conference on World Wide Web, ACM, 2012, pp. 849-858.

[24] K. Moustaghfir, G. Schiuma, M. Mura, E. Lettieri, G. Radaelli and N. Spiller, "Promoting professionals' innovative behaviour through knowledge sharing: the moderating role of social capital", Journal of Knowledge Management, 17 (2013), pp. 527-544.

[25] B. Muthén, "A general structural equation model with dichotomous, ordered categorical, and continuous latent variable indicators", Psychometrika, 49 (1984), pp. 115132.

[26] H. Peng, "Why and when do people hide knowledge?", Journal of Knowledge Management, 17 (2013), pp. 398415.

[27] A. Petróczi, T. Nepusz and F. Bazsó, "Measuring tiestrength in virtual social networks", Connections, 27 (2007), pp. 39-52.

[28] E. M. Steffes and L. E. Burgee, "Social ties and online word of mouth", Internet research, 19 (2009), pp. 42-59.

[29] G. Ver Steeg and A. Galstyan, Information transfer in social media, Proceedings of the 21st international conference on World Wide Web, ACM, 2012, pp. 509-518.

[30] D. Wang, Z. Wen, H. Tong, C.-Y. Lin, C. Song and A.-L. Barabási, Information spreading in context, Proceedings of the 20th international conference on World wide web, ACM, 2011, pp. 735-744.

[31] X. Wang, P. F. Clay and N. Forsgren, "Encouraging knowledge contribution in IT support: social context and the differential effects of motivation type", Journal of Knowledge Management, 19 (2015), pp. 315-333.

[32] J. Wei, B. Bu, X. Guo and M. Gollagher, "The process of crisis information dissemination: impacts of the strength of ties in social networks", Kybernetes, 43 (2014), pp. 178191.
[33] S. Zhou, F. Siu and M. Wang, "Effects of social tie content on knowledge transfer", Journal of Knowledge Management, 14 (2010), pp. 449-463. 